\title{
Brief Comments on Higgs-Boson Discovery Potential of Future Tevatron Running
}

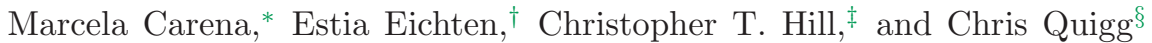 \\ Theoretical Physics Department, Fermi National Accelerator Laboratory, P.O. Box 500, Batavia, Illinois 60510 USA
}

\section{SEARCH FOR THE STANDARD-MODEL HIGGS BOSON}

The standard electroweak theory gives an excellent account of many pieces of data over a wide range of energies. Global fits, made within the framework of the standard electroweak theory, favor a light Higgs boson, and exhibit some tension with direct searches. The LEP experiments, which focused on the $e^{+} e^{-} \rightarrow H Z^{0}$ channel, set a lower bound on the standard-model Higgs-boson mass of $M_{H}>114.4 \mathrm{GeV}$ at $95 \% \mathrm{CL}[1,2]$. The Tevatron experiments CDF and D0 also search for the standardmodel Higgs boson, examining a variety of production channels and decay modes appropriate to different Higgsboson masses. The most recent combined result exclude the range $160 \mathrm{GeV}<M_{H}<170 \mathrm{GeV}$ at $95 \% \mathrm{CL}[3,4]$. See [5] for an overview of past searches [6].

The disjoint exclusion regions from LEP and the Tevatron make it somewhat complicated to specify the remaining mass ranges favored for the standard-model Higgs boson. A useful example is shown in Figure 1 [7]. In the Gfitter analysis, at $2 \sigma$-significance $(\approx 95 \% \mathrm{CL})$, the

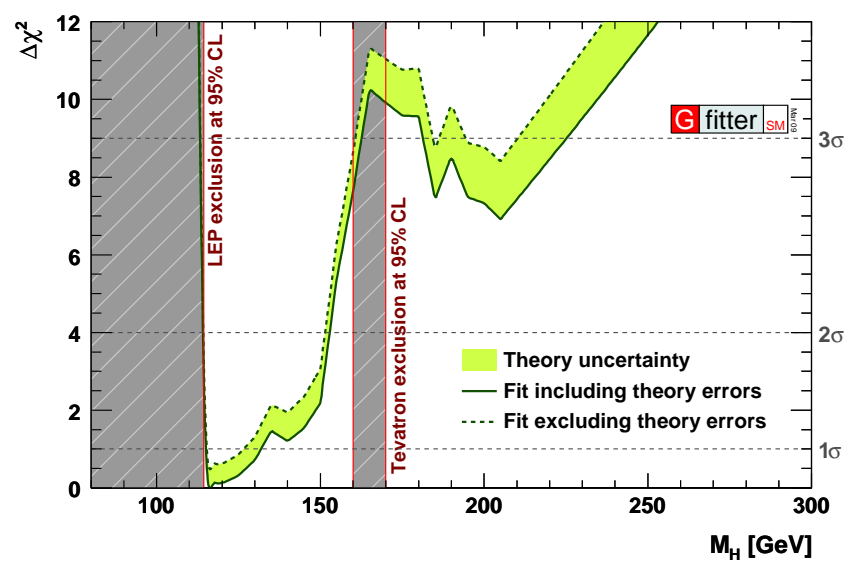

FIG. 1: $\Delta \chi^{2}$ as a function of the Higgs-boson mass for the Gfitter complete fit, taking account of direct searches at LEP and the Tevatron. The solid (dashed) line gives the results when including (ignoring) theoretical errors. The minimum $\Delta \chi^{2}$ of the fit including theoretical errors is used for both curves to obtain the offset-corrected $\Delta \chi^{2}[7]$.

\footnotetext{
*Electronic address: carena@fnal.gov

${ }^{\dagger}$ Electronic address: eichten@fnal.gov

$\ddagger$ Electronic address: hill@fnal.gov

§Electronic address: quigg@fnal.gov
}

standard-model Higgs-boson mass must lie in the interval $113.8 \mathrm{GeV}<M_{H}<152.5 \mathrm{GeV}$. Healthy skepticism dictates that we regard the inferred constraints on $M_{H}$ as a potential test of the standard model, not as rigid boundaries on where the agent of electroweak symmetry breaking must show itself. ${ }^{1}$ Eventually, we must be prepared to search up to $O(1 \mathrm{TeV})$.

The search for the Higgs boson is now the province of the proton accelerators. The $2-\mathrm{TeV}$ proton-antiproton Tevatron Collider is operating now, its integrated luminosity having surpassed $6 \mathrm{fb}^{-1}$, and the $14-\mathrm{TeV}$ Large Hadron Collider at CERN will provide high-luminosity proton-proton collisions beginning in 2009 or 2010.

The Tevatron is expected to operate through 2011, producing a total of $10 \mathrm{fb}^{-1}$ for analysis by the CDF and D0 collaborations. It is worth emphasizing that analysis strategies at the Tevatron are still evolving, becoming more sensitive and more robust, so that the significance of searches may improve more than the growth in statistics. The experimenters are optimistic that a sample of that size will be sufficient - in the absence of a signal-to set a $95 \%$ exclusion limit for the standardmodel Higgs boson for $114 \mathrm{GeV} \lesssim M_{H} \lesssim 190 \mathrm{GeV}$, i.e., over the range currently favored by the global fits [4]. Barring a breakthrough in analysis techniques, discovery of the standard-model Higgs boson at 5- $\sigma$ significance is extremely unlikely at the Tevatron, unless the production rate should be enhanced (for example, by a fourth generation of quarks). At the interesting level of $3-\sigma$ evidence, the situation is more promising. The experiments have quoted the odds of establishing "evidence" at about one in three for $120 \mathrm{GeV} \lesssim M_{H} \lesssim 145 \mathrm{GeV}$, and better than one in two for $M_{H} \lesssim 116 \mathrm{GeV}$ and $150 \mathrm{GeV} \lesssim M_{H} \lesssim 177 \mathrm{GeV}$ [12]. At a minimum, we will know more about where the (standard-model) Higgs boson is not by the time the LHC Higgs search begins in earnest.

An exclusion of the standard-model Higgs boson in the range of masses preferred by the global fits would mean either that the electroweak theory is incomplete or that the analyses have misconstrued the existing data. Without reference to the precision measurements, we can say that if the Higgs-boson mass lies outside the range $134 \mathrm{GeV} \lesssim M_{H} \lesssim 180 \mathrm{GeV}$, then new physics is impli-

\footnotetext{
1 If new strong dynamics - rather than a perturbatively coupled elementary scalar - hides the electroweak symmetry, then the mass of the composite stand-in for the Higgs boson can range up to several hundred $\mathrm{GeV}[8,9]$. The same is true for standard-model fits that allow an extra generation of quarks and leptons $[10,11]$.
} 
cated below the Planck scale $[13,14]$.

\section{POSSIBILITIES BEYOND THE STANDARD-MODEL HIGGS BOSON}

Although the standard electroweak theory correlates many observations, it leaves too many gaps in our understanding for it to be considered a complete theory. We therefore have reason to consider extensions to the standard model, for which the standard-model fits to the electroweak measurements do not apply.

Supersymmetric (SUSY) extensions of the electroweak theory entail considerable model-dependence, but yield high-quality fits to the precision data [15-17]. In the SUSY context, the Higgs search analyses are more complex due to the large number of parameters and new particles. In addition to the lightest SUSY Higgs boson, $h$, there are heavier Higgs bosons (CP-even $H$ and CP-odd $A$ ) and charged Higgs bosons, $H^{ \pm}$. Bounds inferred from searches for the lightest CP-even Higgs boson $h$ of the minimal supersymmetric standard model are somewhat less restrictive than for the standard-model Higgs boson.
The tension between fits that prefer light masses and direct searches that disfavor a light Higgs boson is not present in the supersymmetric world. On the other hand, in its simplest form, the minimal supersymmetric standard model would be challenged if $M_{h}$ exceeded about $135 \mathrm{GeV}$. A recent 25-parameter fit to the "phenomenological minimal supersymmetric standard model" concludes that $117 \mathrm{GeV} \lesssim M_{h} \lesssim 129 \mathrm{GeV}$ at $95 \%$ C.L. [17].

For certain conventional benchmark SUSY parameter choices the Tevatron can completely rule out the minimal supersymmetric standard model (MSSM) at the $95 \%$ confidence level. SUSY cannot be ruled out as a general principle, as small modifications to the MSSM can evade the Higgs discovery limits. The multitude of channels examined at the Tevatron is an asset in the search for SUSY Higgs or general two-Higgs-doublet models, in which signals could pop up more strongly than expected for the standard-model Higgs boson. In any case, a 10$\mathrm{fb}^{-1}$ data set will provide powerful new constraints on supersymmetric models [18]. These constraints would take on added importance, should the Tevatron or LHC uncover evidence for superpartners.
[1] LEP Working Group for Higgs boson searches, R. Barate et al., Phys. Lett. B565, 61-75 (2003) [arXiv:hep-ex/0306033], lephiggs.web.cern.ch.

[2] M. M. Kado and C. G. Tully, Ann. Rev. Nucl. Part. Sci. 52, 65-113 (2002).

[3] Tevatron New Phenomena Higgs Working Group, G. Bernardi et al., "Combined CDF and D0 upper limits on standard model Higgs boson production at high mass $\left(150-200 \mathrm{GeV} / \mathrm{c}^{2}\right)$ with $3 \mathrm{fb}^{-1}$ of data," arXiv:0808.0534 [hep-ex].

[4] Tevatron New Phenomena and Higgs Working Group, "Combined CDF and D0 Upper Limits on Standard Model Higgs-Boson Production with up to $4.2 \mathrm{fb}^{-1}$ of Data," arXiv:0903.4001 [hep-ex], tevnphwg.fnal.gov.

[5] G. Bernardi, M. Carena, and T. Junk, "Higgs bosons: Theory and searches," in [6].

[6] Particle Data Group, C. Amsler et al., Phys. Lett. B667, 1 (2008) pdg. Ibl.gov.

[7] H. Flaecher et al., "Gfitter - Revisiting the Global Electroweak Fit of the Standard Model and Beyond," arXiv:0811.0009 [hep-ph], cern.ch/gfitter.

[8] K. Lane, "Two lectures on technicolor," arXiv: hep-ph/0202255.

[9] C. T. Hill and E. H. Simmons, Phys. Rept. 381, 235 (2003) [arXiv:hep-ph/0203079]. Erratum-ibid. 390, 553 (2004).
[10] G. D. Kribs, T. Plehn, M. Spannowsky, and T. M. P. Tait, Phys. Rev. D76, 075016 (2007) [arXiv:0706.3718 [hep-ph]].

[11] V. A. Novikov, A. N. Rozanov, and M. I. Vysotsky, "Once more on extra quark-lepton generations and precision measurements," arXiv:0904.4570 [hep-ph].

[12] D. Denisov, "Closing in on the Higgs Particle with the Tevatron." Talk at AAAS Annual Meeting, and J. Konigsberg, private communication, 2009.

[13] J. A. Casas, J. R. Espinosa, and M. Quiros, Phys. Lett. B382, 374-382 (1996) [arXiv:hep-ph/9603227].

[14] T. Hambye and K. Riesselmann, Phys. Rev. D55, 7255-7262 (1997) [arXiv:hep-ph/9610272].

[15] J. Erler and D. M. Pierce, Nucl. Phys. B526, 53-80 (1998) [arXiv:hep-ph/9801238].

[16] S. Heinemeyer, W. Hollik, A. M. Weber, and G. Weiglein, JHEP 04, 039 (2008) [arXiv:0710.2972 [hep-ph]].

[17] S. S. AbdusSalam, B. C. Allanach, F. Quevedo, F. Feroz, and M. Hobson, "Fitting the Phenomenological MSSM," arXiv:0904.2548 [hep-ph].

[18] M. Carena, "Supersymmetric Higgs." Talk at SUSY 2009. 